\title{
OPTIMIZATION OF DRX PARAMETER FOR POWER SAVING IN LTE
}

\author{
Usharani.$S^{1}$, Nivetha.$A^{2}$ \\ ${ }^{I} P G$-Scholar, ECE, Manakula Vinayagar Institute of Technology, Pondicherry, India \\ ${ }^{2} P G$-Scholar, ECE, Manakula Vinayagar Institute of Technology, Pondicherry, India
}

\begin{abstract}
Long Term Evolution LTE in mobile communication is mainly implemented for high speed data transmission, but due to large usage of User Equipment (UE) leads to increase in power consumption. As mobile devices increases rapidly the energy consumption becomes an important issue. Discontinuous Reception (DRX) has been implemented so as to reduce the power consumption of the user equipment, and also transmission of large amount of data. In this paper we propose a relative power saving in order to get a precise result, and also the effectiveness of the power saving is done by combining the short DRX and long DRX cycle. Therefore the parameters values are varied to improve the performance of the DRX mechanism which leads to archive larger power saving in User Equipment.
\end{abstract}

Keywords: Relative power saving, power consumption, DRX, LTE.

\section{INTRODUCTION}

In mobile communication there is a increase in large amount of data usage which leads to a development of Long Term of Evolution. LTE is used to provide high spectrum efficiency and large amount of data transmission. In LTE technology the power saving mechanism is achieved by implementing DRX mechanism.DRX is defined as discontinuous reception which allows the user equipment to enter into the power saving mode in order to extend the battery life time of a UE [1].There are many publications on modeling DRX. Example includes UMTS and LTE.

DRX mechanism in UMTS has been studied in [2] and [3] it is based on the Poisson distribution and ETSI bursty traffic model. In UMTS the UE has two parameters they are wake up time and sleep time i.e. the inactive timer and the DRX cycle [3] in sleep period the power is saved by shutting down the RF module. The RF module monitors the physical downlink control channels if any data is received it turns to active state. In IEEE802.16e the mobile station is configured by two DRX modes one is wake up delay and the other is the sleep mode [14]. In wake up period the RF is turned on and the mobile station starts to receive the data from the downlink channel otherwise it enters into the sleep mode this is how the power saved in the mobile station.

In LTE the time period of the inactive time is controlled by the I-timer. There is a possibility of retransmission in this process i.e. the user equipment fails to receive the data in order to avoid this problem the RF module will move to round trip delay time and then it sends the retransmitted data so the accuracy is increased [13].
This paper illustrates the study of the power consumption of the DRX mechanism in long term evolution. The combination of short and long DRX cycle helps to improve the battery life time and reduce the latency produced in the system. Relative power saving is implemented to provide a precise result.

\section{IMPLEMENTATION OF DRX MECHANISM}

In DRX mechanism there exist three mode of operation given as inactive period, short DRX cycle and long DRX cycle which helps in power saving process. The sleep and wake up methods of each User Equipment is determined by four parameters they are DRX short period $\left(\mathrm{t}_{\mathrm{SD}}\right)$, DRX long period $\left(t_{L D}\right)$, DRX inactivity timer $\left(t_{\mathrm{I}}\right)$ and short DRX timer $\left(\mathrm{t}_{\mathrm{N}}\right)$. we focus on the DRX cycle where the UE stay awake during the packet transmission and moves to DRX mode when there is no data transmission. i.e. it enters the power saving mode.

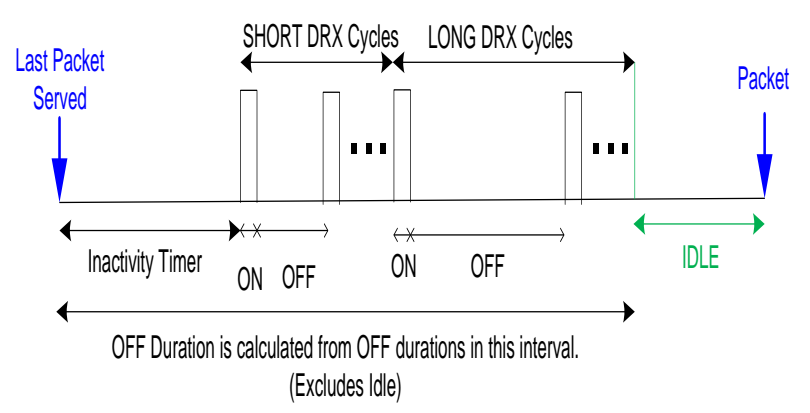

Fig-1: DRX mechanism 


\subsection{Inactive Period}

In this period the UE receives the data from evolved nodeB where the UE enters the active mode. At this state the UE monitors the PDCCH to check whether the data received or not.

Before the expiration of DRX Inactivity timer, if the PDCCH indicates a downlink transmission, another DRX inactive timer is activated or else the expiration of the DRX inactive timer ends the inactivity period. Then the DRX short cycle timer is activated and the short sleep period begins.

\subsection{Short Sleep Period}

Period where the DRX Short Cycle Timer is running Before DRX Short Cycle Timer expires and during On Duration of each DRX Short Cycle, UE wakes up to monitor the $\mathrm{PDCCH}$. If the PDCCH indicates a downlink transmission, UE wakes up, starts DRX Inactivity Timer and transits from the light sleep period into the inactivity period. Otherwise, UE falls asleep until the next On Duration. When DRX Short Cycle Timer expires, the short sleep period ends and the long sleep period follow. During the light sleep period, eNB processor will not transmit any packets to UE.

\subsection{Long Sleep Period}

Period where the UE receiver follows DRX Long Cycle During On Duration of each DRX Long Cycle, UE wakes up to monitor the PDCCH. If the PDCCH indicates a downlink transmission, UE wakes up, starts DRX Inactivity Timer, and transits from the short sleep period into the inactive period. Otherwise, UE falls asleep until the next On Duration. During the long sleep period, eNB processor will not transmit any packets to UE. Under LTE DRX mechanism, the inactive period denotes the power saving mode, while the power saving mode includes the short sleep period and the long sleep period.

\section{MODELING AND ANALYSIS}

The four parameter values which are implemented is defined as following:

The ON duration timer is the time where the DRX cycle begins where the user equipment monitors the physical downlink control channel. The value can range from $1,10,20,100$ to 200 transmission time interval.

The inactive timer denotes the active time mode.

The long DRX period is specified in TTL.

The short DRX period is specified in TTL and can be $2^{\mathrm{x}}$ where $\mathrm{x}=1,2 \ldots 9$ or $5 \mathrm{x} 2^{\mathrm{x}}, \mathrm{p}=1,2 \ldots 6$.
Short DRX cycle timer is denoted by $\mathrm{N}$, in this period no data packets are received and the UE enters the sleep mode.

\subsection{Analysis Process}

The power consumption is denoted as $\mathrm{PC}_{\mathrm{s}}(\mathrm{L})$ and $\mathrm{PC}_{\mathrm{l}}(\mathrm{L})$ for short DRX and long DRX respectively. $\mathrm{L}$ denotes the packet arrival time at the evolved nodeB. The power consumption of short DRX and long DRX is given as

$$
\begin{aligned}
& P C_{S}(L)=L \mathrm{x} P C_{R E}+(O N-L) P C_{I D}+\left(D R X_{S}-O N-1\right) P C_{L S}+P C_{L A} \\
& P C_{l}(L)=L \mathrm{x} P C_{R E}+(O N-L) P C_{I D}+P C_{D L}+P C_{L A}
\end{aligned}
$$

The power consumption in two DRX state is low when compared to active state. In other case there is no power consumption in long DRX mode. The total power consumption can be calculated by counting the number of TTL spends in each state by the user equipment multiplied by the power consumption per each TTL state.

The mean power consumption $E[P C]$ per TTL is given as

$$
E[P C] \frac{P_{S C} P C_{S C}+P_{L C} P C_{L C}}{P_{S C} D R X_{S}+P_{L C} D R X_{l}}
$$

To evaluate the efficiency of the power we are formulating two factor one is the relative power saving factor given as $\rho$ and the other is the relative power saving factor given as $\eta$.The relative power saving factor is the ratio of the mean power consumption of a UE when DRX is enabled to the mean power consumption when DRX is not enabled. It is the actual power saving of a UE and also improves the accuracy of the analytical model without increasing the computational complexity. For precise result we are going for relative power saving instead of power saving factor.

The calculation of $\rho$ involves only the time where the sleep time is equal to the length of the short or long DRX.

$$
\rho=\frac{P_{S C}\left(D R X_{S}-O N\right)+P_{L C}\left(D R X_{l}-O N\right)}{P_{S C}\left(D R X_{S}\right)+P_{L C}\left(D R X_{l}\right)}
$$

The relative power saving is calculated as

$$
\begin{gathered}
\eta=\frac{E\left[P C_{\text {non }-D R X}\right]-E[P C]}{E\left[P C_{\text {non }-D R X}\right]} \\
\eta=1-\frac{E[P C]}{E\left[P C_{\text {non }-D R X}\right]}
\end{gathered}
$$

Where $E[P C]$ is the mean power consumption per TTI and $E\left[P C_{\text {non-DRX }}\right]$ is the mean power consumption when DRX is not enabled. During $E\left[P C_{n o n-D R X}\right]$ calculation there is no need of considering the sleep state but the active state is taken under 
consideration. By using the above equation we can able to calculate the relative power saving factor.

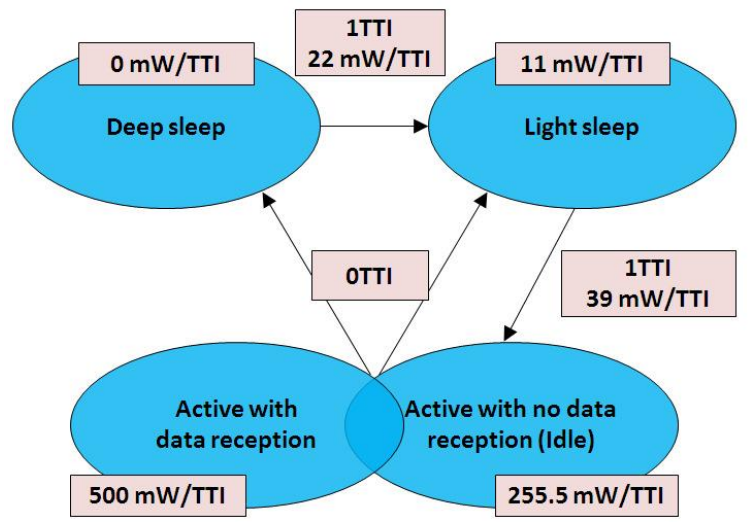

Fig 2: power consumption of UE in different state

The power consumption in Long and short DRX are smaller when compared with active mode.

\section{SIMULATION RESULT}

The mean arrival rate on power consumption is first investigated the power consumption is plotted related to the arrival time this helps to examine the power consumed under different traffic state. Greater the arrival rate value leads to the high power, this makes the UE not to enter the long DRX cycle hence greater power consumption is obtained.

Next to notice the short DRX cycle where the short DRX timer $\mathrm{N}$ increases the probability for the UE to enter into the next state which is the long DRX cycle. The figure 3 and 4 indicates the short DRX cycle of power saving factor and relative power saving.

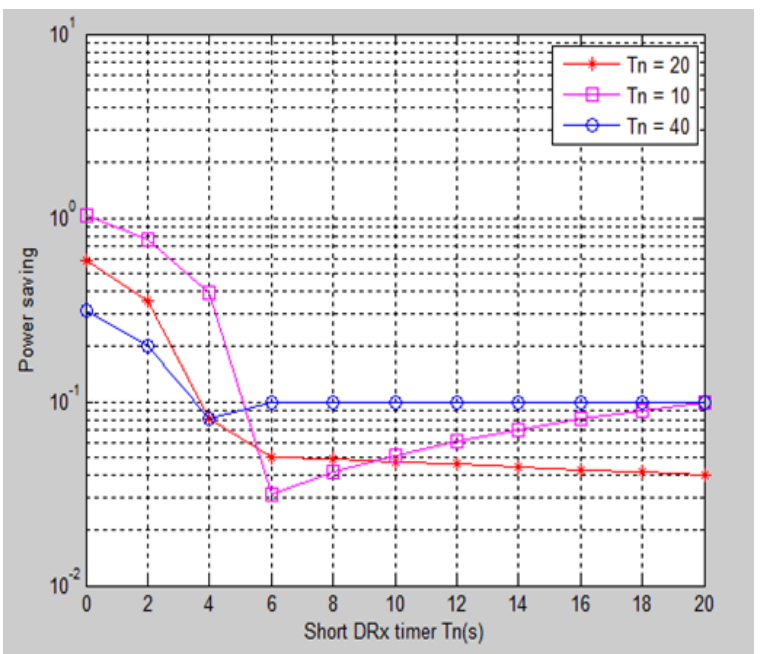

Fig 3: short DRX cycle for power saving factor

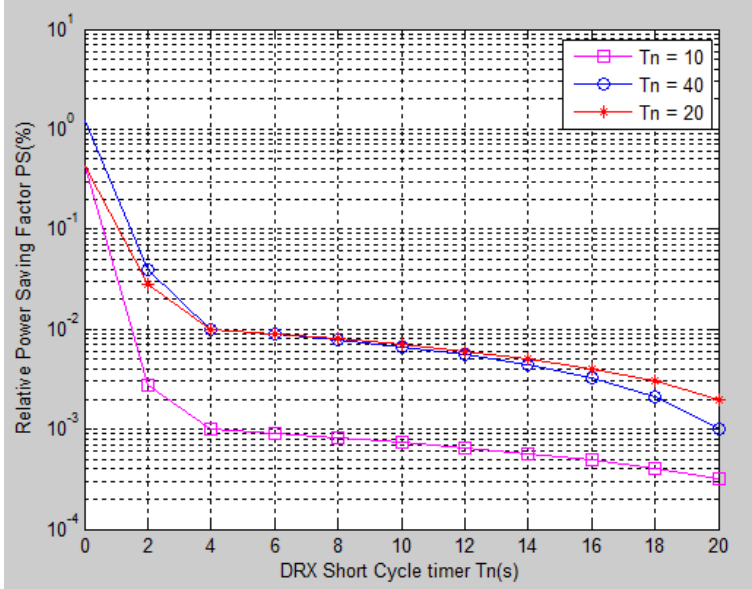

Fig 4: short DRX cycle for relative power saving factor

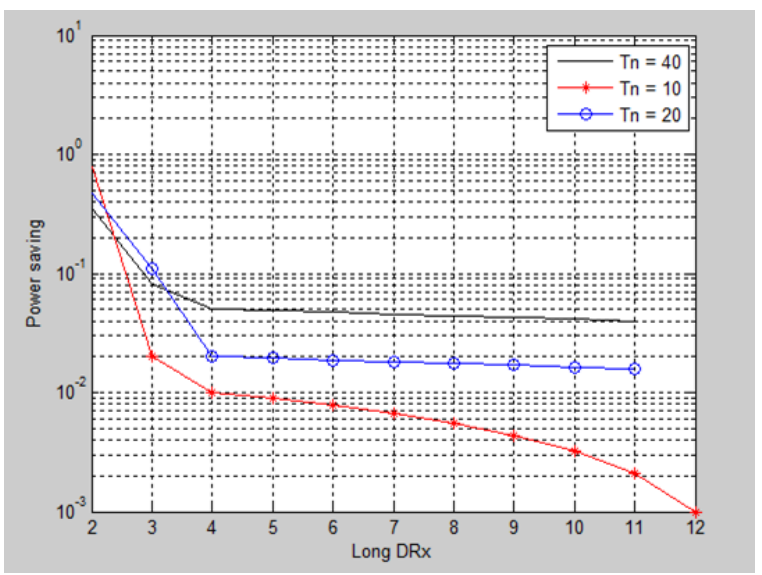

Fig 5: long DRX cycle for power saving factor

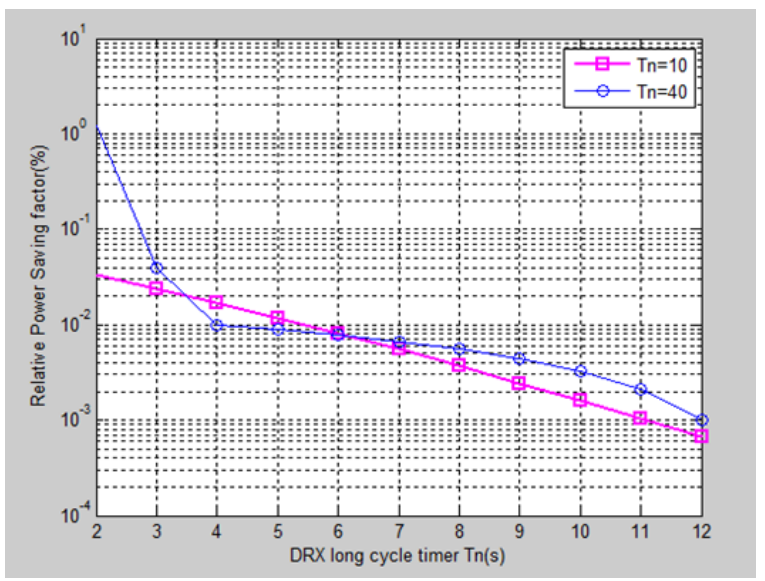

Fig 6: long DRX cycle for relative power saving factor

The figure 5 and 6 indicates the long DRX cycle for power saving factor and relative power saving factor. In long DRX the curve indicates length coverage. The power saving is high in long DRX cycle. Smaller the short DRX value increases the 
long DRX value hence the long DRX helps to achieve the maximum power saving. The proper selection of the parameter helps to achieve power saving above 90 percent.

\section{CONCLUSIONS}

The effectiveness and accurate analysis of the power saving is increased by the mixture of short and long DRX cycle. The accuracy is obtained by introducing the relative power saving factor. In simulation part the parameters values are varied according to their standard, by varying the parameter value we also investigate the performance standard. The proper selection of the parameter helps to increase the power conservation.

\section{ACKNOWLEDGEMENTS}

I wish to thank Mr. S.Arunmozhi, HOD of Electronics and Communication, for his wonderful support to do this research. Next I would thank Mrs.K.priethamje vithya our guide, for her splendid effort in completion of this research work. I would like to convey special thanks to all my colleagues for encouraging me in bringing up this work to an extinct level

\section{REFERENCES}

[1]. 3GPP TS36.300 V9.2.0. Evolved Universal Terrestrial Radio Access (EUTRA) and Evolved Universal Terrestrial Radio Access Network (EUTRAN); Overall Description; Stage 2, Dec. 2009.

[2]. S. R. Yang and Y. B. Lin, "Modeling UMTS discontinuous reception mechanism," IEEE Trans. Wireless Comm., vol. 4, no. 1, pp. 312-319, Jan. 2005.

[3]. S. R. Yang, S. Y. Yan, and H. N. Hung, "Modeling UMTS power saving with bursty packet data traffic," IEEE Trans. Mob. Comp., vol. 6, no. 12, pp. 1398-1409, 2007

[4]. T. E. Kolding, J. Wigard, and L. Dalsgaard, "Balancing power saving and single user experience with discontinuous reception in LTE," Proc. ISWCS'08, Reykjavik, October 2008.

[5]. Steepest Ascent, 3G Evolution Lab-LTE Toolbox2010. http://www.steepestascent.com/content/mediaassets/html/LTE /Help/ PDCCH\%20Decoding\%20Example.html

[6]. C. S. Bontu and E. Illidge, "DRX mechanism for power saving in LTE," IEEE Comm. Mag., pp. 48-55, June 2009.

[7]. H. C. Wang, C. C. Tseng, G. Y. Chen, F. C. Kuo, and K. C. Ting, "Accurate analysis of delay and power consumption of LTE DRX mechanism with a combination of short and long cycles," in Proc. $15^{\text {th }}$ International Symposium on Wireless Personal Multimedia Communications (WPMC), pp. 384-388, Taipei, Taiwan, Sept. 24-27, 2012.

[8]. Kuo-Chang Ting, Hwang-Cheng Wang, Chih-Cheng Tseng, and Fang- Chang Kuo, "Energy-Efficient DRX Scheduling for QoS Traffic in LTE Networks," in Proc. IEEE International Symposium on Parallel and Distributed
Processing with Applications, pp. 213-218, Busan, Korea, May 26-28, 2011.

[9]. D. Vinella and M. Polignano, Discontinuous Reception and Transmission (DRX/DTX) Strategies in Long Term Evolution (LTE) for Voice-over-IP Traffic under both FullDynamic and Semi-Persistent Packet Scheduling Policies, Master Thesis, Aalborg University, 2009.

[10]. Nokia, DRX Parameters in LTE, March 2007.

[11]. Nokia, Evaluating DRX Concepts for E-UTRAN, March 2007.

[12]. L. Zhou, et al., "Performance analysis of power saving mechanism with adjustable DRX cycles in 3GPP LTE," in Proc. IEEE 68th Vehicular Technology Conference (VTC 2008-Fall), Sept. 21-24, 2008.

[13]. Y. Y. Mihov, K. M. Kassev, and B. P. Tsankov, "Analysis and performance evaluation of the DRX mechanism for power saving in LTE," in Proc. IEEE 26th Electrical and Electronics Engineers in Israel, pp. 520-524, Nov. 17-20, 2010.

[14]. S. Yang, M. Yoo, and Y. Shin, "Adaptive discontinuous reception mechanism for power saving in UMTS," IEEE Comm. Lett., vol. 11, no. 1, pp. 40-42, Jan. 2007.

[15]. Y. Xiao, "Energy saving mechanism in the IEEE 802.16e wireless MAN," IEEE Comm. Lett., pp. 595-597, July 2005.

[16]. 3GPP TS 36.321, E-UTRA; Medium Access Control (MAC) Protocol Specification, v. 8.7.0, Sept. 2009.

[17]. K. Aho, J. Puttonen, T. Henttonen, and L. Dalsgaard, "Channel quality indicator preamble for discontinuous reception," in Proc. IEEE Vehicular Technology ConferenceSpring, pp. 1-5, May 2010.

[18]. M. Demirhan, A. T. Koc, S. Shrivastava, and R. Vannithamby, Adaptive DRX Cycle Length Based on Available Battery Power, US Patent number 20070291673 A1, Dec. 2007.

\section{BIOGRAPHIES}

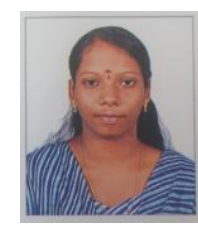

S. Usharani received her B.Tech in ECE degree from Mailam engineering college, Anna University in the year 2012. She is pursuing her M.Tech in ECE from Manakula Vinayagar Institute of Technology, Pondicherry University.

E-mail: ushaece91@gmail.com

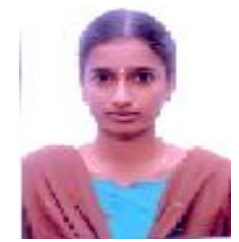

A. Nivetha received her B.Tech in ECE degree from SKP engineering College thiruvanamalai in the year 2012.she is pursuing her M.Tech in ECE from Manakula Vinayagar Institute of Technology, Pondicherry University

E-mail:nivethaadhi@gmail.com 